\title{
Abundance and Impact of Doubly Charged Polyatomic Argon Interferences in ICPMS Spectra
}

Bodo Hattendorf ${ }^{1 *}$, Bianca Gusmini ${ }^{1}$, Ladina Dorta ${ }^{1}$, Robert S. Houk ${ }^{2}$, Detlef Günther ${ }^{1}$

1: ETH Zurich, Department of Chemistry and Applied Biosciences, Laboratory for Inorganic Chemistry, Vladimir Prelog Weg 1, 8093 Zurich, Switzerland

2: Ames Laboratory U. S. Department of Energy, Department of Chemistry, Iowa State University, Ames IA 50011, USA

*email: bodo@inorg.chem.ethz.ch

\section{Supplementary Information}

Table S1: Full list of isotopes potentially affected by spectral interference from $\mathrm{MAr}^{2+}$ ions including $\mathrm{m} / \mathrm{Q}$ relative abundance (in\%) and mass resolving power required to separate the two.

\begin{tabular}{|c|c|c|c|c|c|}
\hline Analyte & Isotope & $\mathrm{MAr}^{2+}$ & $\mathrm{m} / \mathrm{Q}$ & $\begin{array}{c}\text { Relative } \\
\text { Abundance, \% }\end{array}$ & $\mathrm{m} / \Delta \mathrm{m}$ \\
\hline $\mathrm{Si}$ & 30 & $24 \mathrm{Mg} 36 \mathrm{Ar}$ & 29.97602 & 0.27 & 13331 \\
\hline$P$ & 31 & $24 \mathrm{Mg} 38 \mathrm{Ar}$ & 30.97361 & 0.049 & 207182 \\
\hline$P$ & 31 & $26 \mathrm{Mg} 36 \mathrm{Ar}$ & 30.9748 & 0.038 & 29999 \\
\hline$S$ & 32 & $26 \mathrm{Mg} 38 \mathrm{Ar}$ & 31.97239 & 0.007 & 101018 \\
\hline S & 32 & 24Mg40Ar & 31.97344 & 78.6 & 23388 \\
\hline$S$ & 33 & $26 \mathrm{Mg} 40 \mathrm{Ar}$ & 32.97221 & 11.1 & 43671 \\
\hline K & 39 & 40Ca38Ar & 38.96239 & 0.061 & 29484 \\
\hline K & 39 & 42Ca36Ar & 38.96281 & 0.0022 & 43341 \\
\hline $\mathrm{Ca}$ & 40 & 42Ca38Ar & 39.9604 & 0.00041 & 18256 \\
\hline $\mathrm{Ca}$ & 40 & 44Ca36Ar & 39.96124 & 0.0071 & 29591 \\
\hline $\mathrm{Ca}$ & 40 & 40Ca40Ar & 39.96221 & 96.6 & 105442 \\
\hline K & 41 & 44Ca38Ar & 40.95883 & 0.0013 & 13693 \\
\hline K & 41 & 46Ti36Ar & 40.95981 & 0.027 & 20374 \\
\hline $\mathrm{K}$ & 41 & $42 \mathrm{Ca} 40 \mathrm{Ar}$ & 40.96023 & 0.65 & 25641 \\
\hline K & 41 & 46Ca36Ar & 40.96034 & 0.000014 & 27630 \\
\hline $\mathrm{Ca}$ & 42 & 46Ti38Ar & 41.95741 & 0.005 & 34548 \\
\hline $\mathrm{Ca}$ & 42 & 48Ti36Ar & 41.95747 & 0.25 & 36470 \\
\hline $\mathrm{Ca}$ & 42 & 46Ca38Ar & 41.95794 & 0.0000025 & 61120 \\
\hline $\mathrm{Ca}$ & 42 & 44Ca40Ar & 41.95866 & 2.1 & 1134017 \\
\hline $\mathrm{Ca}$ & 42 & 48Ca36Ar & 41.95976 & 0.00065 & 36741 \\
\hline $\mathrm{Ca}$ & 43 & 48Ti38Ar & 42.95506 & 0.046 & 11593 \\
\hline $\mathrm{Ca}$ & 43 & 50Ti36Ar & 42.95589 & 0.018 & 14921 \\
\hline $\mathrm{Ca}$ & 43 & $50 \mathrm{~V} 36 \mathrm{Ar}$ & 42.95708 & 0.00085 & 25397 \\
\hline $\mathrm{Ca}$ & 43 & 46Ti40Ar & 42.95723 & 7.9 & 27950 \\
\hline $\mathrm{Ca}$ & 43 & 48Ca38Ar & 42.95736 & 0.00012 & 30403 \\
\hline $\mathrm{Ca}$ & 43 & 46Ca40Ar & 42.95776 & 0.004 & 42576 \\
\hline $\mathrm{Ca}$ & 44 & 50Ti38Ar & 43.95348 & 0.0034 & 21967 \\
\hline
\end{tabular}




\begin{tabular}{|c|c|c|c|c|c|}
\hline Analyte & Isotope & $\mathrm{MAr}^{2+}$ & $\mathrm{m} / \mathrm{Q}$ & $\begin{array}{c}\text { Relative } \\
\text { Abundance, \% }\end{array}$ & $\mathrm{m} / \Delta \mathrm{m}$ \\
\hline $\mathrm{Ca}$ & 44 & $50 \mathrm{~V} 38 \mathrm{Ar}$ & 43.95467 & 0.00016 & 54033 \\
\hline $\mathrm{Ca}$ & 44 & 48Ti40Ar & 43.95489 & 73.5 & 73875 \\
\hline $\mathrm{Ca}$ & 44 & 48Ca40Ar & 43.95718 & 0.19 & 25894 \\
\hline Sc & 45 & 50Ti40Ar & 44.95331 & 5.4 & 17261 \\
\hline Sc & 45 & 50V40Ar & 44.9545 & 0.25 & 31726 \\
\hline $\mathrm{Ni}$ & 60 & $84 \mathrm{Sr} 36 \mathrm{Ar}$ & 59.94021 & 0.0019 & 6360 \\
\hline $\mathrm{Ni}$ & 61 & $84 \mathrm{Sr} 38 \mathrm{Ar}$ & 60.93781 & 0.00035 & 9032 \\
\hline $\mathrm{Ni}$ & 61 & $86 \mathrm{Sr} 36 \mathrm{Ar}$ & 60.93813 & 0.034 & 8612 \\
\hline $\mathrm{Ni}$ & 62 & $86 \mathrm{Sr} 38 \mathrm{Ar}$ & 61.93573 & 0.0062 & 8390 \\
\hline $\mathrm{Ni}$ & 62 & $88 \mathrm{Sr} 36 \mathrm{Ar}$ & 61.93631 & 0.28 & 7776 \\
\hline $\mathrm{Ni}$ & 62 & $84 \mathrm{Sr} 40 \mathrm{Ar}$ & 61.93763 & 0.56 & 6670 \\
\hline $\mathrm{Cu}$ & 63 & $88 \mathrm{Sr} 38 \mathrm{Ar}$ & 62.9339 & 0.052 & 14619 \\
\hline $\mathrm{Cu}$ & 63 & $86 \mathrm{Sr} 40 \mathrm{Ar}$ & 62.93555 & 9.82 & 10569 \\
\hline $\mathrm{Cu}$ & 63 & $90 \mathrm{Zr} 36 \mathrm{Ar}$ & 62.93585 & 0.17 & 10064 \\
\hline $\mathrm{Zn}$ & 64 & $90 \mathrm{Zr} 38 \mathrm{Ar}$ & 63.93345 & 0.032 & 14867 \\
\hline $\mathrm{Zn}$ & 64 & $88 \mathrm{Sr} 40 \mathrm{Ar}$ & 63.93373 & 82.2 & 13946 \\
\hline $\mathrm{Zn}$ & 64 & $92 \mathrm{Zr} 36 \mathrm{Ar}$ & 63.93602 & 0.058 & 9302 \\
\hline $\mathrm{Cu}$ & 65 & $90 \mathrm{Zr} 40 \mathrm{Ar}$ & 64.93327 & 51.2 & 11851 \\
\hline $\mathrm{Cu}$ & 65 & $92 \mathrm{Zr} 38 \mathrm{Ar}$ & 64.93361 & 0.011 & 11159 \\
\hline $\mathrm{Cu}$ & 65 & $94 \mathrm{Zr} 36 \mathrm{Ar}$ & 64.93666 & 0.059 & 7324 \\
\hline $\mathrm{Zn}$ & 66 & $92 \mathrm{Zr} 40 \mathrm{Ar}$ & 65.93344 & 17.1 & 8908 \\
\hline $\mathrm{Zn}$ & 66 & $94 \mathrm{Zr} 38 \mathrm{Ar}$ & 65.93425 & 0.011 & 8025 \\
\hline $\mathrm{Zn}$ & 66 & $96 \mathrm{Zr} 36 \mathrm{Ar}$ & 65.93763 & 0.0095 & 5684 \\
\hline $\mathrm{Zn}$ & 67 & $94 \mathrm{Zr} 40 \mathrm{Ar}$ & 66.93408 & 17.3 & 9634 \\
\hline $\mathrm{Zn}$ & 67 & $96 \mathrm{Zr} 38 \mathrm{Ar}$ & 66.93523 & 0.0018 & 8265 \\
\hline $\mathrm{Zn}$ & 68 & $96 \mathrm{Zr} 40 \mathrm{Ar}$ & 67.93505 & 2.77 & 6655 \\
\hline Se & 74 & $112 \mathrm{Sn} 36 \mathrm{Ar}$ & 73.93553 & 0.0033 & 5664 \\
\hline As & 75 & $112 \mathrm{Sn} 38 \mathrm{Ar}$ & 74.93312 & 0.00061 & 6501 \\
\hline As & 75 & $114 \operatorname{Sn} 36 \mathrm{Ar}$ & 74.93489 & 0.0022 & 5636 \\
\hline $\mathrm{Se}$ & 76 & $114 \mathrm{Sn} 38 \mathrm{Ar}$ & 75.93248 & 0.00041 & 5719 \\
\hline Se & 76 & $112 \mathrm{Sn} 40 \mathrm{Ar}$ & 75.93295 & 0.97 & 5525 \\
\hline Se & 76 & $116 \mathrm{Sn} 36 \mathrm{Ar}$ & 75.93437 & 0.049 & 5007 \\
\hline Se & 77 & $116 \mathrm{Sn} 38 \mathrm{Ar}$ & 76.93196 & 0.0093 & 6381 \\
\hline $\mathrm{Se}$ & 77 & $114 \mathrm{Sn} 40 \mathrm{Ar}$ & 76.93231 & 0.65 & 6203 \\
\hline $\mathrm{Se}$ & 77 & $118 \mathrm{Sn} 36 \mathrm{Ar}$ & 76.9343 & 0.083 & 5344 \\
\hline Se & 78 & $116 \mathrm{Sn} 40 \mathrm{Ar}$ & 77.93179 & 14.6 & 5379 \\
\hline $\mathrm{Se}$ & 78 & $118 \mathrm{Sn} 38 \mathrm{Ar}$ & 77.93189 & 0.015 & 5340 \\
\hline Se & 78 & $120 \mathrm{Sn} 36 \mathrm{Ar}$ & 77.9346 & 0.11 & 4506 \\
\hline $\mathrm{Br}$ & 79 & $118 \mathrm{Sn} 40 \mathrm{Ar}$ & 78.93172 & 24.2 & 5896 \\
\hline $\mathrm{Br}$ & 79 & $120 \mathrm{Sn} 38 \mathrm{Ar}$ & 78.93219 & 0.02 & 5696 \\
\hline $\mathrm{Br}$ & 79 & $122 \mathrm{Sn} 36 \mathrm{Ar}$ & 78.93522 & 0.016 & 4675 \\
\hline $\mathrm{Se}$ & 80 & $120 \mathrm{Sn} 40 \mathrm{Ar}$ & 79.93202 & 32.3 & 5158 \\
\hline Se & 80 & $122 \mathrm{Sn} 38 \mathrm{Ar}$ & 79.93281 & 0.0029 & 4906 \\
\hline Se & 80 & $124 \mathrm{Sn} 36 \mathrm{Ar}$ & 79.93613 & 0.019 & 4075 \\
\hline $\mathrm{Br}$ & 81 & $122 \mathrm{Sn} 40 \mathrm{Ar}$ & 80.93264 & 4.6 & 4950 \\
\hline
\end{tabular}




\begin{tabular}{|c|c|c|c|c|c|}
\hline Analyte & Isotope & $\mathrm{MAr}^{2+}$ & $\mathrm{m} / \mathrm{Q}$ & $\begin{array}{c}\text { Relative } \\
\text { Abundance, \% }\end{array}$ & $\mathrm{m} / \Delta \mathrm{m}$ \\
\hline $\mathrm{Br}$ & 81 & $124 \mathrm{Sn} 38 \mathrm{Ar}$ & 80.93373 & 0.0035 & 4641 \\
\hline Se & 82 & 124Sn40Ar & 81.93355 & 5.6 & 4864 \\
\hline $\mathrm{Kr}$ & 83 & 130Ba36Ar & 82.93664 & 0.00037 & 3685 \\
\hline $\mathrm{Sr}$ & 84 & 130Ba38Ar & 83.93423 & 0.000069 & 4034 \\
\hline $\mathrm{Sr}$ & 84 & 132Ba36Ar & 83.93602 & 0.00034 & 3714 \\
\hline $\mathrm{Rb}$ & 85 & 132Ba38Ar & 84.93361 & 0.000063 & 3893 \\
\hline $\mathrm{Rb}$ & 85 & 130Ba40Ar & 84.93406 & 0.11 & 3815 \\
\hline $\mathrm{Rb}$ & 85 & 134Ba36Ar & 84.93574 & 0.0082 & 3546 \\
\hline $\mathrm{Sr}$ & 86 & 134Ba38Ar & 85.93334 & 0.0015 & 3570 \\
\hline $\mathrm{Sr}$ & 86 & 132Ba40Ar & 85.93344 & 0.099 & 3555 \\
\hline $\mathrm{Sr}$ & 86 & 136Ba36Ar & 85.93578 & 0.027 & 3241 \\
\hline $\mathrm{Sr}$ & 86 & 136Ce36Ar & 85.93707 & 0.00065 & 3091 \\
\hline $\mathrm{Sr}$ & 87 & 134Ba40Ar & 86.93316 & 2.4 & 3582 \\
\hline $\mathrm{Rb}$ & 87 & 136Ba38Ar & 86.93337 & 0.0049 & 3594 \\
\hline $\mathrm{Sr}$ & 87 & 136Ce38Ar & 86.93466 & 0.00012 & 3374 \\
\hline $\mathrm{Sr}$ & 87 & 138Ba36Ar & 86.93612 & 0.24 & 3194 \\
\hline $\mathrm{Sr}$ & 87 & 138Ce36Ar & 86.9365 & 0.00085 & 3150 \\
\hline $\mathrm{Sr}$ & 87 & 138La36Ar & 86.93706 & 0.00031 & 3087 \\
\hline $\mathrm{Sr}$ & 88 & 136Ba40Ar & 87.93319 & 7.8 & 3189 \\
\hline $\mathrm{Sr}$ & 88 & 138Ba38Ar & 87.93371 & 0.045 & 3130 \\
\hline $\mathrm{Sr}$ & 88 & $138 \mathrm{Ce} 38 \mathrm{Ar}$ & 87.93409 & 0.00016 & 3088 \\
\hline $\mathrm{Sr}$ & 88 & 136Ce40Ar & 87.93449 & 0.19 & 3046 \\
\hline $\mathrm{Sr}$ & 88 & 138La38Ar & 87.93465 & 0.000057 & 3029 \\
\hline $\mathrm{Sr}$ & 88 & $140 \mathrm{Ce} 36 \mathrm{Ar}$ & 87.93622 & 0.3 & 2873 \\
\hline$Y$ & 89 & 138Ba40Ar & 88.93353 & 71.4 & 3212 \\
\hline$Y$ & 89 & $140 \mathrm{Ce} 38 \mathrm{Ar}$ & 88.93381 & 0.056 & 3180 \\
\hline$Y$ & 89 & 138Ce40Ar & 88.93391 & 0.25 & 3169 \\
\hline$Y$ & 89 & 138La40Ar & 88.93447 & 0.09 & 3107 \\
\hline$Y$ & 89 & $142 \mathrm{Nd} 36 \mathrm{Ar}$ & 88.93736 & 0.092 & 2822 \\
\hline$Y$ & 89 & $142 \mathrm{Ce} 36 \mathrm{Ar}$ & 88.93812 & 0.038 & 2755 \\
\hline $\mathrm{Zr}$ & 90 & 140Ce40Ar & 89.93364 & 88.1 & 3108 \\
\hline $\mathrm{Zr}$ & 90 & $142 \mathrm{Nd} 38 \mathrm{Ar}$ & 89.93496 & 0.017 & 2972 \\
\hline $\mathrm{Zr}$ & 90 & $142 \mathrm{Ce} 38 \mathrm{Ar}$ & 89.93572 & 0.007 & 2899 \\
\hline $\mathrm{Zr}$ & 90 & $144 \mathrm{Nd} 36 \mathrm{Ar}$ & 89.93855 & 0.081 & 2657 \\
\hline $\mathrm{Zr}$ & 90 & $144 \mathrm{Sm} 36 \mathrm{Ar}$ & 89.9395 & 0.011 & 2584 \\
\hline $\mathrm{Zr}$ & 91 & $142 \mathrm{Nd} 40 \mathrm{Ar}$ & 90.93478 & 27 & 3120 \\
\hline $\mathrm{Zr}$ & 91 & $142 \mathrm{Ce} 40 \mathrm{Ar}$ & 90.93554 & 11 & 3041 \\
\hline $\mathrm{Zr}$ & 91 & $144 \mathrm{Nd} 38 \mathrm{Ar}$ & 90.93614 & 0.015 & 2981 \\
\hline $\mathrm{Zr}$ & 91 & 144Sm38Ar & 90.9371 & 0.0019 & 2890 \\
\hline $\mathrm{Zr}$ & 91 & $146 \mathrm{Nd} 36 \mathrm{Ar}$ & 90.94006 & 0.058 & 2641 \\
\hline $\mathrm{Zr}$ & 92 & $144 \mathrm{Nd} 40 \mathrm{Ar}$ & 91.93596 & 23.7 & 2972 \\
\hline $\mathrm{Zr}$ & 92 & 144Sm40Ar & 91.93692 & 3.1 & 2883 \\
\hline $\mathrm{Zr}$ & 92 & $146 \mathrm{Nd} 38 \mathrm{Ar}$ & 91.93765 & 0.011 & 2818 \\
\hline $\mathrm{Zr}$ & 92 & $148 \mathrm{Sm} 36 \mathrm{Ar}$ & 91.94091 & 0.038 & 2562 \\
\hline $\mathrm{Zr}$ & 92 & $148 \mathrm{Nd} 36 \mathrm{Ar}$ & 91.94195 & 0.02 & 2490 \\
\hline
\end{tabular}




\begin{tabular}{|c|c|c|c|c|c|}
\hline Analyte & Isotope & $\mathrm{MAr}^{2+}$ & $\mathrm{m} / \mathrm{Q}$ & $\begin{array}{c}\text { Relative } \\
\text { Abundance, \% }\end{array}$ & $\mathrm{m} / \Delta \mathrm{m}$ \\
\hline $\mathrm{Nb}$ & 93 & $146 \mathrm{Nd} 40 \mathrm{Ar}$ & 92.93748 & 17.1 & 2987 \\
\hline $\mathrm{Nb}$ & 93 & $148 \mathrm{Sm} 38 \mathrm{Ar}$ & 92.93851 & 0.0071 & 2892 \\
\hline $\mathrm{Nb}$ & 93 & $148 \mathrm{Nd} 38 \mathrm{Ar}$ & 92.93954 & 0.0036 & 2801 \\
\hline $\mathrm{Nb}$ & 93 & 150Sm36Ar & 92.94214 & 0.025 & 2598 \\
\hline $\mathrm{Nb}$ & 93 & $150 \mathrm{Nd} 36 \mathrm{Ar}$ & 92.94395 & 0.019 & 2473 \\
\hline $\mathrm{Zr}$ & 94 & 148Sm40Ar & 93.93833 & 11.3 & 2933 \\
\hline $\mathrm{Zr}$ & 94 & $148 \mathrm{Nd} 40 \mathrm{Ar}$ & 93.93937 & 5.7 & 2842 \\
\hline $\mathrm{Zr}$ & 94 & 150Sm38Ar & 93.93973 & 0.0047 & 2810 \\
\hline $\mathrm{Zr}$ & 94 & $150 \mathrm{Nd} 38 \mathrm{Ar}$ & 93.94154 & 0.0036 & 2666 \\
\hline $\mathrm{Zr}$ & 94 & 152Sm36Ar & 93.94337 & 0.087 & 2535 \\
\hline Mo & 94 & 152Gd36Ar & 93.9434 & 0.00068 & 2451 \\
\hline Mo & 95 & 150Sm40Ar & 94.93956 & 7.4 & 2814 \\
\hline Mo & 95 & 152Sm38Ar & 94.94096 & 0.016 & 2702 \\
\hline Mo & 95 & $152 \mathrm{Gd} 38 \mathrm{Ar}$ & 94.94099 & 0.00013 & 2700 \\
\hline Mo & 95 & $150 \mathrm{Nd} 40 \mathrm{Ar}$ & 94.94137 & 5.6 & 2671 \\
\hline Mo & 95 & $15 \mathrm{Gd} 36 \mathrm{Ar}$ & 94.94394 & 0.0074 & 2491 \\
\hline Mo & 95 & 154Sm36Ar & 94.94461 & 0.077 & 2448 \\
\hline Mo & 96 & 152Sm40Ar & 95.94079 & 25.6 & 2656 \\
\hline Mo & 96 & $152 \mathrm{Gd} 40 \mathrm{Ar}$ & 95.94082 & 0.2 & 2654 \\
\hline Mo & 96 & $15 \mathrm{Gd} 38 \mathrm{Ar}$ & 95.94153 & 0.0014 & 2602 \\
\hline $\mathrm{Zr}$ & 96 & 154Sm38Ar & 95.9422 & 0.014 & 2827 \\
\hline Mo & 96 & $156 \mathrm{Gd} 36 \mathrm{Ar}$ & 95.94456 & 0.07 & 2404 \\
\hline $\mathrm{Ru}$ & 96 & 156Dy36Ar & 95.94564 & 0.0002 & 2521 \\
\hline Mo & 97 & $15 \mathrm{Gd} 40 \mathrm{Ar}$ & 96.94135 & 2.2 & 2742 \\
\hline Mo & 97 & 154Sm40Ar & 96.94203 & 22.6 & 2691 \\
\hline Mo & 97 & $156 \mathrm{Gd} 38 \mathrm{Ar}$ & 96.94216 & 0.013 & 2682 \\
\hline Mo & 97 & 156Dy38Ar & 96.94323 & 0.000038 & 2604 \\
\hline Mo & 97 & $158 \mathrm{Gd} 36 \mathrm{Ar}$ & 96.94555 & 0.084 & 2451 \\
\hline Mo & 97 & 158Dy36Ar & 96.9457 & 0.00034 & 2442 \\
\hline Mo & 98 & 156Gd40Ar & 97.94198 & 20.4 & 2677 \\
\hline Mo & 98 & 156Dy40Ar & 97.94306 & 0.06 & 2600 \\
\hline Mo & 98 & $158 \mathrm{Gd} 38 \mathrm{Ar}$ & 97.94315 & 0.016 & 2594 \\
\hline $\mathrm{Ru}$ & 98 & 158Dy38Ar & 97.9433 & 0.000063 & 2576 \\
\hline $\mathrm{Ru}$ & 98 & 160Dy36Ar & 97.9461 & 0.008 & 2399 \\
\hline Mo & 98 & $160 \mathrm{Gd} 36 \mathrm{Ar}$ & 97.94703 & 0.074 & 2352 \\
\hline $\mathrm{Ru}$ & 99 & 158Gd40Ar & 98.94297 & 24.7 & 2671 \\
\hline $\mathrm{Ru}$ & 99 & 158Dy40Ar & 98.94312 & 0.1 & 2660 \\
\hline $\mathrm{Ru}$ & 99 & 160Dy38Ar & 98.94369 & 0.0015 & 2620 \\
\hline $\mathrm{Ru}$ & 99 & $160 \mathrm{Gd} 38 \mathrm{Ar}$ & 98.94462 & 0.014 & 2557 \\
\hline $\mathrm{Ru}$ & 99 & 162Dy36Ar & 98.9469 & 0.087 & 2414 \\
\hline $\mathrm{Ru}$ & 99 & $162 \mathrm{Er} 36 \mathrm{Ar}$ & 98.94789 & 0.00048 & 2357 \\
\hline $\mathrm{Ru}$ & 100 & 160Dy40Ar & 99.94352 & 2.3 & 2542 \\
\hline $\mathrm{Ru}$ & 100 & 160Gd40Ar & 99.94445 & 21.8 & 2483 \\
\hline Mo & 100 & 162Dy38Ar & 99.94449 & 0.016 & 2699 \\
\hline $\mathrm{Ru}$ & 100 & $162 \mathrm{Er} 38 \mathrm{Ar}$ & 99.94548 & 0.000088 & 2421 \\
\hline
\end{tabular}




\begin{tabular}{|c|c|c|c|c|c|}
\hline Analyte & Isotope & $\mathrm{MAr}^{2+}$ & $\mathrm{m} / \mathrm{Q}$ & $\begin{array}{c}\text { Relative } \\
\text { Abundance, \% }\end{array}$ & $\mathrm{m} / \Delta \mathrm{m}$ \\
\hline $\mathrm{Ru}$ & 100 & 164Dy36Ar & 99.94809 & 0.096 & 2277 \\
\hline $\mathrm{Ru}$ & 100 & 164Er36Ar & 99.9481 & 0.0055 & 2276 \\
\hline $\mathrm{Ru}$ & 101 & 162Dy40Ar & 100.9443 & 25.4 & 2605 \\
\hline $\mathrm{Ru}$ & 101 & 162Er40Ar & 100.9453 & 0.14 & 2540 \\
\hline $\mathrm{Ru}$ & 101 & 164Dy38Ar & 100.9457 & 0.018 & 2516 \\
\hline $\mathrm{Ru}$ & 101 & 164Er38Ar & 100.9457 & 0.001 & 2515 \\
\hline $\mathrm{Ru}$ & 101 & 166Er36Ar & 100.9487 & 0.11 & 2343 \\
\hline $\mathrm{Ru}$ & 102 & 164Dy40Ar & 101.9455 & 28 & 2475 \\
\hline $\mathrm{Ru}$ & 102 & 164Er40Ar & 101.9455 & 1.6 & 2474 \\
\hline $\mathrm{Ru}$ & 102 & 166Er38Ar & 101.9462 & 0.021 & 2432 \\
\hline $\mathrm{Ru}$ & 102 & 168Er36Ar & 101.9497 & 0.091 & 2247 \\
\hline $\mathrm{Ru}$ & 102 & $168 \mathrm{Yb} 36 \mathrm{Ar}$ & 101.9505 & 0.00044 & 2210 \\
\hline $\mathrm{Rh}$ & 103 & 166Er40Ar & 102.9461 & 33.5 & 2537 \\
\hline $\mathrm{Rh}$ & 103 & 168Er38Ar & 102.9473 & 0.017 & 2463 \\
\hline $\mathrm{Rh}$ & 103 & $168 \mathrm{Yb} 38 \mathrm{Ar}$ & 102.948 & 0.000082 & 2419 \\
\hline $\mathrm{Rh}$ & 103 & $170 \mathrm{Yb} 36 \mathrm{Ar}$ & 102.9509 & 0.01 & 2268 \\
\hline $\mathrm{Rh}$ & 103 & 170Er36Ar & 102.9512 & 0.051 & 2250 \\
\hline $\mathrm{Ru}$ & 104 & 168Er40Ar & 103.9471 & 26.7 & 2493 \\
\hline $\mathrm{Ru}$ & 104 & $168 \mathrm{Yb} 40 \mathrm{Ar}$ & 103.9479 & 0.13 & 2448 \\
\hline $\mathrm{Ru}$ & 104 & $170 \mathrm{Yb} 38 \mathrm{Ar}$ & 103.9485 & 0.0019 & 2413 \\
\hline $\mathrm{Ru}$ & 104 & $170 \mathrm{Er} 38 \mathrm{Ar}$ & 103.9488 & 0.0094 & 2394 \\
\hline $\mathrm{Pd}$ & 104 & $172 \mathrm{Yb} 36 \mathrm{Ar}$ & 103.9517 & 0.075 & 2180 \\
\hline $\mathrm{Pd}$ & 105 & $170 \mathrm{Yb} 40 \mathrm{Ar}$ & 104.9483 & 3 & 2427 \\
\hline $\mathrm{Pd}$ & 105 & 170Er40Ar & 104.9487 & 14.8 & 2407 \\
\hline $\mathrm{Pd}$ & 105 & $172 \mathrm{Yb} 38 \mathrm{Ar}$ & 104.9493 & 0.014 & 2373 \\
\hline $\mathrm{Pd}$ & 105 & $174 \mathrm{Yb} 36 \mathrm{Ar}$ & 104.9529 & 0.11 & 2192 \\
\hline $\mathrm{Pd}$ & 105 & $174 \mathrm{Hf} 36 \mathrm{Ar}$ & 104.9535 & 0.00054 & 2165 \\
\hline $\mathrm{Pd}$ & 106 & $172 \mathrm{Yb} 40 \mathrm{Ar}$ & 105.9491 & 21.8 & 2321 \\
\hline $\mathrm{Pd}$ & 106 & $174 \mathrm{Yb} 38 \mathrm{Ar}$ & 105.9505 & 0.02 & 2251 \\
\hline $\mathrm{Pd}$ & 106 & $174 \mathrm{Hf} 38 \mathrm{Ar}$ & 105.9511 & 0.0001 & 2223 \\
\hline $\mathrm{Pd}$ & 106 & $176 \mathrm{Hf} 36 \mathrm{Ar}$ & 105.9542 & 0.018 & 2087 \\
\hline $\mathrm{Pd}$ & 106 & $176 \mathrm{Yb} 36 \mathrm{Ar}$ & 105.9548 & 0.043 & 2064 \\
\hline $\mathrm{Pd}$ & 106 & 176Lu36Ar & 105.9548 & 0.0088 & 2062 \\
\hline $\mathrm{Ag}$ & 107 & $174 \mathrm{Yb} 40 \mathrm{Ar}$ & 106.9504 & 31.7 & 2362 \\
\hline $\mathrm{Ag}$ & 107 & $174 \mathrm{Hf} 40 \mathrm{Ar}$ & 106.9509 & 0.16 & 2331 \\
\hline $\mathrm{Ag}$ & 107 & $176 \mathrm{Hf} 38 \mathrm{Ar}$ & 106.9518 & 0.0033 & 2289 \\
\hline $\mathrm{Ag}$ & 107 & $176 \mathrm{Yb} 38 \mathrm{Ar}$ & 106.9524 & 0.008 & 2261 \\
\hline $\mathrm{Ag}$ & 107 & 176Lu38Ar & 106.9524 & 0.0016 & 2258 \\
\hline $\mathrm{Ag}$ & 107 & $178 \mathrm{Hf} 36 \mathrm{Ar}$ & 106.9554 & 0.092 & 2127 \\
\hline $\mathrm{Pd}$ & 108 & $176 \mathrm{Hf} 40 \mathrm{Ar}$ & 107.9516 & 5.2 & 2261 \\
\hline $\mathrm{Pd}$ & 108 & $176 \mathrm{Yb} 40 \mathrm{Ar}$ & 107.9522 & 12.6 & 2234 \\
\hline $\mathrm{Pd}$ & 108 & 176Lu40Ar & 107.9523 & 2.6 & 2231 \\
\hline $\mathrm{Pd}$ & 108 & $178 \mathrm{Hf} 38 \mathrm{Ar}$ & 107.9529 & 0.017 & 2200 \\
\hline $\mathrm{Pd}$ & 108 & $180 \mathrm{Hf} 36 \mathrm{Ar}$ & 107.9568 & 0.12 & 2040 \\
\hline $\mathrm{Pd}$ & 108 & 180Ta36Ar & 107.9572 & 0.000041 & 2023 \\
\hline
\end{tabular}




\begin{tabular}{|c|c|c|c|c|c|}
\hline Analyte & Isotope & $\mathrm{MAr}^{2+}$ & $\mathrm{m} / \mathrm{Q}$ & $\begin{array}{c}\text { Relative } \\
\text { Abundance, \% }\end{array}$ & $\mathrm{m} / \Delta \mathrm{m}$ \\
\hline $\mathrm{Ag}$ & 109 & $178 \mathrm{Hf} 40 \mathrm{Ar}$ & 108.9528 & 27 & 2268 \\
\hline $\mathrm{Ag}$ & 109 & $180 \mathrm{Hf} 38 \mathrm{Ar}$ & 108.9544 & 0.022 & 2195 \\
\hline $\mathrm{Ag}$ & 109 & 180Ta38Ar & 108.9548 & 0.0000076 & 2175 \\
\hline $\mathrm{Pd}$ & 110 & $180 \mathrm{Hf} 40 \mathrm{Ar}$ & 109.9542 & 35.1 & 2242 \\
\hline $\mathrm{Pd}$ & 110 & 180Ta40Ar & 109.9547 & 0.012 & 2221 \\
\hline Sn & 120 & 204Pb36Ar & 119.97 & 0.0048 & 1768 \\
\hline $\mathrm{Sb}$ & 121 & 204Pb38Ar & 120.9676 & 0.00088 & 1895 \\
\hline $\mathrm{Sb}$ & 121 & 206Pb36Ar & 120.9707 & 0.082 & 1807 \\
\hline Sn & 122 & 204Pb40Ar & 121.9674 & 1.4 & 1905 \\
\hline Sn & 122 & 206Pb38Ar & 121.9683 & 0.015 & 1879 \\
\hline Sn & 122 & 208Pb36Ar & 121.9718 & 0.18 & 1783 \\
\hline $\mathrm{Sb}$ & 123 & 206Pb40Ar & 122.9681 & 24 & 1923 \\
\hline $\mathrm{Sb}$ & 123 & 208Pb38Ar & 122.9694 & 0.033 & 1885 \\
\hline Sn & 124 & 208Pb40Ar & 123.9692 & 52.2 & 1937 \\
\hline $\mathrm{Ba}$ & 134 & 232Th36Ar & 134.0025 & 0.34 & 1366 \\
\hline $\mathrm{Ba}$ & 135 & 232Th38Ar & 135.0001 & 0.063 & 1428 \\
\hline $\mathrm{Ba}$ & 135 & $234 \mathrm{U} 36 \mathrm{Ar}$ & 135.004 & 0.00002 & 1372 \\
\hline $\mathrm{Ba}$ & 136 & 232Th40Ar & 135.9999 & 99.6 & 1425 \\
\hline $\mathrm{Ba}$ & 136 & 234U38Ar & 136.0016 & 0.0000038 & 1401 \\
\hline $\mathrm{Ba}$ & 137 & $234 \mathrm{U} 40 \mathrm{Ar}$ & 137.0014 & 0.006 & 1432 \\
\hline $\mathrm{Ba}$ & 137 & $238 \mathrm{U} 36 \mathrm{Ar}$ & 137.0089 & 0.34 & 1328 \\
\hline $\mathrm{Ba}$ & 138 & 238U38Ar & 138.0065 & 0.063 & 1362 \\
\hline La & 139 & $238 \mathrm{U} 40 \mathrm{Ar}$ & 139.0063 & 98.9 & 1390 \\
\hline
\end{tabular}

